\title{
Développement De La Méthode De Français Basé Sur Google Classroom En Classe XI Au SMA Negeri 21 Medan
}

\author{
Nuriaty Prasetya Lubis, Junita Friska, Zulherman \\ Program Studi Magister Pendidikan Bahasa Prancis, Universitas Negeri Medan \\ Jalan Willem Iskandar, Pasar V, Medan Estate, 20221, Sumatera Utara, Indonesia \\ E-mail: nuriaty36688107@gmail.com
}

\begin{abstract}
RÉSUMÉ
Cette recherche est la recherche de développement en utilisant la méthode de Borg \& Gall (2005) qui vise à déterminer le processus et l'efficacité du media E-learning basés sur Google Classroom en classe XI, SMA Negeri 21 Medan. La méthode utilisée pour analyser les données est une technique qualitative. Toutes les données recueillies ont été analysées à l'aide de techniques statistiques descriptives qui ont été exprimées dans la distribution des scores et des catégories d'échelle. Le processus d'apprentissage en utilisant le Google Classroom en Classe XI à SMA Negeri 21 Medan, à savoir : la phase d'analyse, phase de conception, phase de développement, phase de mise en œuvre et phase d'évaluation. Les résultats de la validation par le validateur en matériaux est la valeur moyenne 77,95 \% (bien) et la valeur du média d'apprentissage de Google Classroom est 77, $72 \%$, (bien). Ensuite description de données des résultats des tests d'utilisation se compose de données sur les résultats du test d'utilisation des médias par l'enseignant. La moyenne est $81.06 \%$ (très bien). Les données sur le résultat de l'utilisation des médias par les lycéens se compose du test individuel est la moyenne $87,3 \%$ (très bien), le test en petit groupe est la moyenne $89 \%$ (très bien), le test d'essai limité est la moyenne 91,7 \% (très bien). La valeur moyenne des résultats d'apprentissage avec l'apprentissage utilisant les médias d'apprentissage de Google Classroom a obtenu le score le plus élevé au post-test de 100,00, meilleur que le score le plus élevé au pré-test de 77,00.
\end{abstract}

Mots-clés : E-learning, Google Classroom, L'apprentissage De Français.

\section{INTRODUCTION}

Le gouvernement procède les modifications du curriculum afin d'obtenir les meilleurs résultats. Le curriculum est un élément qui contribue à concrétiser le processus de développement de la qualité de ce potentiel. Le curriculum de KTSP, par exemple, il est préparé par le gouvernement en impliquant la communauté et les enseignants afin que le programme de formation soit organisé en fonction des conditions réelles dans l'école. Le gouvernement est en train de créer un nouveau programme appelé le curriculum
2013, basé sur la compétence en tant qu'instrument permettant aux étudiants de devenir: (1) des humains qualifiés qui sont capables de réagir de manière proactive aux défis d'une époque; (2) les personnes éduquées qui croient et craignent le Dieu, noble, en bonne santé, informé, créatif, indépendant; et (3) des citoyens démocratiques et responsables, comme indiqué dans le module de formation sur la mise en œuvre du curriculum de 2013.

Pour atteindre la qualité telle que définie dans le document de curriculum de 2013, les activités d'apprentissage doivent 


\section{Développement De La Méthode De Français Basé Sur Google Classroom \\ En Classe XI Au SMA Negeri 21 Medan}

utiliser des principes centrés sur les lycéens, développer leur créativité, créer des conditions amusantes et stimulantes, et des valeurs contenues telles que l'éthique, l'esthétique, la logique et la kinesthésie, tout en apportant une expérience supplémentaire. Apprentissage diversifié grâce à l'application de diverses stratégies et méthodes d'apprentissage amusantes, contextuelles, efficaces, efficientes et significatives. Lors d'une leçon, l'enseignant fournit aux étudiants une expérience d'apprentissage leur permettant de s'impliquer activement dans la réalisation de diverses activités susceptibles de les aider à développer leur potentiel pour devenir une compétence, comme indiqué dans le document de programme de 2013.

SMA N 21 Medan est l'une des écoles secondaires qui dispose de bonnes installations d'apprentissage. Parmi les installations et infrastructures disponibles pour soutenir des résultats d'apprentissage maximum. Cela est prouvé par plusieurs installations d'apprentissage qui prennent en charge le processus d'apprentissage, notamment la disponibilité d'Infocus, de laboratoires informatiques et de réseaux wifi disponibles pour aider les enseignants à trouver de nouvelles connaissances. Mais en réalité, les installations disponibles n'encouragent pas les résultats d'apprentissage des élèves. Cela prouve clairement de la note moyenne encore faible. Des élèves de la classe XI qui n'a pas' encore atteint le KKM (critère d'exhaustivité minimale). KKM pour les sujets de langue française est de 75, tandis que la valeur moyenne des résultats des élèves de classe XI est 69,83. Cela signifie que les résultats d'apprentissage n'ont pas été maximisés. Pour mettre en œuvre l'apprentissage, une stratégie d'apprentissage appropriée est nécessaire. Les stratégies utilisées dans l'apprentissage fourniront la preuve que l'apprentissage sera plus efficace dans l'apprentissage du français. Développement de matériel pédagogique visant à stimuler la mémoire et la motivation pours apprendre du matériel tiré des cours de français.

Le matériel pédagogique joue un rôle important dans la détermination du niveau d'apprentissage des élèves. Les matériels d'enseignement occupent une place centrale pour trois raisons: 1) en tant que représentation des offres d'enseignants, 2) en tant que moyen d'atteindre les normes de compétences et 3) en tant qu'optimisation des services aux étudiants. Comme décrit ci-après: (1) du matériel didactique en tant que représentation matérielle de l'explication de l'enseignant devant la classe. Les information, les descriptions qui doivent être soumises et les information qui doivent être présentées par l'enseignant sont rassemblées dans le matériel pédagogique. Ainsi, les enseignants peuvent réduire les activités pour expliquer la leçon, afin de disposer de suffisamment de temps pour guider les lycéens dans l'apprentissage ou l'apprentissage des élèves. (2) les matériels d'enseignement en tant qu'outil ou moyen d'atteindre les compétences de base et les compétences de base. Par conséquent, la préparation du matériel didactique est guidée par la compétence de contenu (KI) et la compétence de base (KD) contenues dans les normes de contenu énoncées dans le Règlement ministériel numéro 22 de 2006. (3) le matériel pédagogique est également une forme de service d'unité éducative destiné aux étudiants. Les lycéens s'occupent du matériel documenté et des informati cohérentes afin que les lycéens puissent optimiser leurs capacités en apprenant le matériel d'enseignement de manière répétée. Ainsi, l'optimisation des services d'apprentissage pour les lycéens peut être maintenue grâce à l'utilisation de matériel pédagogique.

Le développement de la technologie est également très influent sur le monde de l'éducation. Les

établissements d'enseignement qui ne mettent pas en œuvre la technologie, en particulier les ordinateurs, ne seront pas compétitifs. L'utilisation des ordinateurs dans les écoles est un exemple d'école qui améliore la qualité de ses institutions.

Parce que ces outils permettent aux écoles d'accroître l'accès, d'accélérer le processus et de réduire l'administration bureaucratique conventionnelle. Avec ce développement technologique rapide, il influence assez la structure de la vie humaine dans tous les secteurs. 
Maintenant, les écoles sont initiées à l'utilisation des technologies de l'information et de la communication (TIK). Avec l'utilisation des ordinateurs dans l'apprentissage, les technologies de l'information et de la communication jouent au moins trois rôles: le contenu de l'apprentissage, les supports d'apprentissage et les outils d'apprentissage. L'une des utilisations des TIK dans l'éducation est l'utilisation de l'apprentissage e-learning en tant que support d'apprentissage. L'apprentissage elearning est un système d'apprentissage qui utilise les médias électroniques comme un outil d'aide aux activités d'apprentissage.

L'utilisation de l'apprentissage elearning est une solution au manque d'accès à l'éducation. Pour que les citoyens puissent bénéficier d'une éducation, qu'elle soit personnelle (classique) ou moderne, l'une d'entre elles est réalisée par l'Internet. Soekartawi (2007, p.4) a dit la même chose: "...l'apprentissage e-learning n'est en effet très utile pour résoudre les problèmes d'éducation impliquant des efforts pour améliorer l'équité et l'accès à l'éducation".

Apprentissage sur le Web qui est souvent utilisé aujourd'hui, comme Schoology, Moodle, Edmodo, Google Classroom et bien d'autres. La technologie Internet offrant un espace et un temps interactifs, flexibles et illimités devrait constituer l'un des meilleurs moyens d'apprentissage. Mais en réalité, l'utilisation de la technologie comme moyen d'apprentissage reste minime. L'enseignant n'utilise toujours pas les installations existantes de manière optimale. Les lycéens n'utilisent que des livres imprimés, travaillent au tableau et utilisent des feuilles de travail.

Google Classroom est une porte qui s'adresse à tous les types d'enseignement et vise à trouver une solution au problème de la réalisation, du partage et du classement de chaque devoir sans papier. L'utilisation de Google Classroom en classe peut se faire par le biais de multiplateformes, notamment par un ordinateur. Les enseignants et les élèves peuvent visiter le site https://classroom.google.com ou télécharger l'application sur Android ou par l'App Store sur iOS avec le mot clé Google
Classroom. Les installations disponibles dans e-learning basé sur Google Classroom pour soutenir l'apprentissage sont nombreuses, mais en réalité, les enseignants qui utilisent des installations informatiques connectées à Internet pour des activités d'apprentissage sont encore très peu. Lors de l'apprentissage chez SMA Negeri 21 Medan, la matière française sont souvent considérées comme moins intéressantes que d'autres matières, ce qui entraîne une faible motivation pour apprendre le français à l'école. Les lycéens doivent se concentrer et porter une attention particulière au matériel présenté lors des activités d'apprentissage. Mais en réalité, certains étudiants hésitent encore à faire attention au matériel fourni par l'enseignant. Pour améliorer la qualité de l'apprentissage pour le mieux, il est nécessaire de disposer d'un support d'apprentissage capable de favoriser l'esprit d'apprentissage et l'intérêt des élèves pour les supports permettant d'optimiser le processus d'apprentissage du français dans SMA Negeri 21 Medan.

\section{MÉTHODOLOGIE DE RECHERCHE}

Cette recherche est fait à SMAN 21 Medan aux élèves de la classe XI, l'année scolaire $2020 / 2021$. Les étapes de cette recherche utilisent 2 étapes, à savoir la première étape pour le développement des médias dans la production de produits et la seconde étape teste le produit pour voir l'efficacité de l'apprentissage. La méthode de recherche utilisée est la recherche et le développement (R \& D) car cette recherche inclut la recherche sur le développement de l'éducation qui vise à produire des produits d'apprentissage pouvant être utilisés et nécessaires, selon les besoins. Borg \& Gall (2005) fixe des limites à la recherche pour le développement dans le but de développer et valider les produits utilisés dans l'éducation.

Les procédures de développement sont divisées en 5 étapes, à savoir: (Borg \& Gall : 2005) : (1) la première étape de la recherche préliminaire, cette étape commence par l'identification des besoins d'apprentissage et la détermination des normes de compétence du sujet, l'analyse de l'apprentissage, les caractéristiques 
Comportement initial de l'élève, Déterminer les compétences de base et les indicateurs, Rédiger des tests de référence, Élaborer des stratégies d'apprentissage, Élaborer du matériel pédagogique (2) La deuxième étape de la conception de médias, Cette deuxième étape commence par la conception de logiciels, la fabrication de manuscrits, le scénarimage, la création d' organigrammes voir (3) la troisième étape de la collecte de matériel, en commençant par la collecte de matériel pour la fabrication et la collecte d'images animées, l'enregistrement et la collecte d'auditions (4) la quatrième étape de fabrication et l'apprentissage e-learning de matières françaises à l'aide de la schoologie, qui est complétée par des instructions aux médias comme: instructions d'apprentissage, description du manioc, compétences de base, description du matériel, questions relatives à la pratique et retours d'information, la clôture est un résumé et (5) la cinquième étape de la révision par des experts et des tests dans le contexte de l'évaluation formative et de la révision du produit. L'évaluation formative se poursuit au cours du processus de \développement, depuis l'analyse, la conception de la production et la mise en œuvre jusqu'aux résultats obtenus conformément aux objectifs fixés.

\section{RÉSULTATS ET DISCUSSION}

Les Réponses des questionnaires d'analyse des besoins "Le Média d'apprentissage E-Learning sur Google Classroom dans l'apprentissage de Français.

Tableau 1. Questionnaire de Recherche

\begin{tabular}{c|c|c|c|c}
\hline \multirow{2}{*}{ No } & $\begin{array}{c}\text { Les } \\
\text { questionnaires }\end{array}$ & Oui & Non & $\begin{array}{c}\text { Peut- } \\
\text { être }\end{array}$ \\
\cline { 3 - 5 } 1. & $\begin{array}{c}\text { Apprendre en } \\
\text { utilisant les } \\
\text { médias est plus } \\
\text { amusant que } \\
\text { d'utiliser } \\
\text { seuelement des } \\
\text { méthodes } \\
\text { conventionnelles } \\
\text { (conférences). }\end{array}$ & $100 \%$ & - & - \\
\hline 2. & $\begin{array}{c}\text { Je suis plus en } \\
\text { mesure de bien }\end{array}$ & $89,5 \%$ & $5 \%$ & $5 \%$ \\
\hline
\end{tabular}

\begin{tabular}{|c|c|c|c|c|}
\hline & $\begin{array}{c}\text { suivre la leçon sur } \\
\text { ce que } \\
\text { l'enseignant } \\
\text { explique lors de } \\
\text { l'utilisation des } \\
\text { médias que les } \\
\text { cours magistraux. }\end{array}$ & & & \\
\hline 3. & $\begin{array}{l}\text { Je m'ennuie } \\
\text { rapidement } \\
\text { lorsque je } \\
\text { participe à des } \\
\text { cours qui juste } \\
\text { écrire et écouter } \\
\text { sans interaction } \\
\text { directe. }\end{array}$ & $90 \%$ & $5 \%$ & $5 \%$ \\
\hline 4. & $\begin{array}{c}\text { Apprendre } \\
\text { quelque chose } \\
\text { avec de méthodes } \\
\text { conventionnelles } \\
\text { (cours } \\
\text { magistraux) } \\
\text { m'ennuie plus } \\
\text { rapidement et j'ai } \\
\text { tendance à } \\
\text { préférer interagir } \\
\text { avec des amis } \\
\text { plutôt que de } \\
\text { prêter attention } \\
\text { au matériel } \\
\text { présenté. }\end{array}$ & $70 \%$ & $15 \%$ & $15 \%$ \\
\hline 5. & $\begin{array}{c}\text { Je préfère un } \\
\text { processus } \\
\text { d'apprentissage } \\
\text { interactif } \\
\text { (discussion, } \\
\text { utiliser de } \\
\text { méthodes } \\
\text { intéressantes, } \\
\text { utiliser de } \\
\text { supports des jeux, } \\
\text { etc) parce que je } \\
\text { peux comprendre } \\
\text { le sujet } \\
\text { d'apprentissage } \\
\text { plus rapidement }\end{array}$ & $80 \%$ & $5 \%$ & $15 \%$ \\
\hline 6. & $\begin{array}{c}\text { Vous avez un } \\
\text { Android/tablette/ } \\
\text { ordinateur } \\
\text { portable/ordinate } \\
\text { ur. }\end{array}$ & $95 \%$ & - & $5 \%$ \\
\hline 7. & $\begin{array}{c}\text { Voua avez } \\
\text { entendu des } \\
\text { supports } \\
\text { d'apprentissage } \\
\text { comme e-learning }\end{array}$ & $30 \%$ & $55 \%$ & $15 \%$ \\
\hline 8. & $\begin{array}{c}\text { Vous connaissez } \\
\text { de l'application } \\
\text { google class. }\end{array}$ & $95 \%$ & - & $5 \%$ \\
\hline
\end{tabular}




\begin{tabular}{|c|c|c|c|c|}
\hline 9. & $\begin{array}{l}\text { Vous avez utilisé } \\
\text { l'application } \\
\text { Google Classroom } \\
\text { comme support } \\
\text { d'apprentissage } \\
\text { pour les matières } \\
\text { à l'école. }\end{array}$ & $90 \%$ & - & $10 \%$ \\
\hline 10. & $\begin{array}{l}\text { Vous comprenez } \\
\text { les fonctionnalités } \\
\text { de l'application } \\
\text { Google Classroom. }\end{array}$ & $50 \%$ & $10 \%$ & $40 \%$ \\
\hline
\end{tabular}

Processus de développement du Méthode Français basé sur Google Classroom Le processus de développement Google Classroom se déroule par 5 étapes. Les étapes suivies sont les suivantes:

a. Phase d'Analyse (Analyse)

Cette étape est effectuée une analyse pour obtenir des information relatives aux médias d'apprentissage en fonction de ce qui est nécessaire par les étudiants. Ensuite, cette analyse a été menée en observant des étudiants du troisième semestre du programme d'étude de la langue française à l'Univésité de Medan. Les données des étudiants ont été collectées en distribuant des questionnaires pour analyser les besoins des médias d'apprentissage via. Cette collecte de données montre les besoins d'un média d'apprentissage sous la forme Google Classroom matériel pédagogique développé sous la forme Google Classroom.

\section{b. Phase de Conception (Design)}

La phase de conception est la prochaine étape de la phase d'analyse. À ce stade, l'auteur détermine des objectifs pédagogiques tels que la détermination des compétences de base, des objectifs et des descriptions. Après cela, l'auteur détermine également les étapes de la séquence média à développer, c'est-àdire qu'il détermine le concept de média Google Classroom.

c. Phase de Développement (Development) Cette étape de développement est une étape importante du processus de création de média Google Classroom parce que la phase de développement est un composant multimédia qui sera utilisé pour créer des médias, tels que l'ajustement de l'mage, de la vidéo avec les matériaux d'apprentissage, c'est-à dire, Passé Composé, Message courts et annonces.

d. Phase de Mise en œuvre (Implémentation)

A la phase de mise en œuvre, les résultats du développement des médias l'étape précédente sont immédiatement réalisés afin de connaître l'état de média google classroom lorsqu'ils sont exploités.

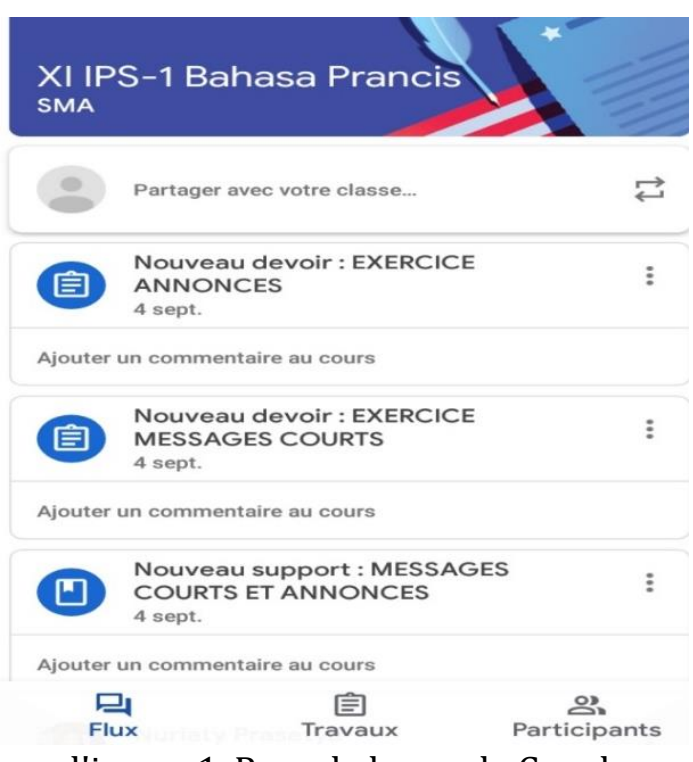

l'image 1. Page de home de Google Classroom

e. Phase d'Évaluation (Evaluation)

L'étape d'évaluation est l'étape d'évaluation à chaque étape du développement et pas seulement le produit final. Après avoir traversé uncertain nombre d'étapes, les médias Google Classroom méritent d'être présentés dans le processus d'apprentissage.

f. Résultats de Validité du Multimédia d'Apprentissage

Les résultats de la faisabilité de développer des supports pédagogiques basés sur la validation du produit à travers une série d'essais et de révisions qui ont été effectués, il est constaté que les résultats du développement de 
supports interactifs sur le matériau de texte descriptif ont été utilisés. Les résultats de validité du développement de média d'apprentissage et d'essais sont réalisés grâce à la validation d'experts en matériau de langue française et à la validation d'experts en supports d'apprentissage. La validation du produit a pour but de connaître l'opinion d'experts du matériau et des supports d'apprentissage sur la conception de l'apprentissage, les aspects de la présentation de l'apprentissage et la densité du contenu des supports d'apprentissage. Les catégories utilisées dans cette validation sont : très décent (4), digne (3), inéligible (2), très inadéquate (1).

Résultats de Validité du Multimédia d'Apprentissage Validation par l'Expert en Matériau

L'évaluation effectuée par l'expert en matériau comprenait des aspects de l'évaluation de la pertinence du contenu, de la pertinence de la présentation, de la pertinence de la langue et des graphiques des supports d'apprentissage Google Classroom. On examine les résultats de validation obtenus. Le score total est 77, 95 $\%$ (Bien).

\section{Validation par l'Expert en Média}

Les résultats de la validation par d'expert en média sur le Google Classroom sous les quatre aspects indiquent que Google Classroom peut être utilisé dans l'apprentissage décrire un lieu. Cela ressort des résultats de la validation sur les quatre aspects de l'évaluation avec une moyenne de $77,72 \%$ qui est dans la catégorie des «bien».

Description des données des résultats des tests d'utilisation de Média.

1. Données sur les résultats du test d'utilisation des médias par l'enseignant.

Basé sur les résultats du test d'utilisation des médias par l'enseignant ci-dessus, on peut voir que les médias développés sont passés par le processus de révision et le test d'utilisation des médias par l'enseignant dans l'essai limité des médias développés an obtenu le score totals de 81,06\% dans la catégorie Très bien.

a. Données sur les résultats de l'utilisation des médias par les lycéens

1. Le test individuel

Les résultats de des tests d'utilisation des Médias avec test individuel à SMA Negeri 21 Medan, une moyenne de $87,3 \%$ qui est dans la catégorie de « Très bien».

2. Le test en petit groupe

Les résultats de des tests d'utilisation des Médias par les petits essais à SMA Negeri 21 Medan, une moyenne de $89 \%$ qui est dans la catégorie de "Très bien».

3. Le test d'essai limité

Les résultats de des tests d'utilisation des Médias par les essais limités à SMA Negeri 21 Medan, une moyenne de 91, $7 \%$ qui est dans la catégorie de « Très bien».

Description des données de recherche

a. Analyse des résultats des données de recherche

Cette recherche a fait dans la classe XI IPS-1 SMA Negeri 21 Medan située à Jalan Selambo Ujung, kelurahan Medan Tenggara, Kecamatan Medan Denai. La première étape prise dans une classe est de donner aux Lycéens un prétest pour savoir les capacités initiales de chaque lycéen. Ensuite, dans l'apprentissage de Google Classroom. Avec le matériel Passe Composé et Messages Courts et Annonces. À la fin du processus d'apprentissage, un postest sera donné pour déterminer les résultats d'apprentissage des lycéens. Sur la base de la recherche, les résultats du test initial (pré-test) et du test final (posttest) ont été obtenus pour la classe composée de 32 étudiants. Après cela, les calculs sont effectués de manière à obtenir la moyenne du prétest, du posttest et de l'écart type comme dans le tableau 2. 
Tableau 2. Statistiques donnés Pre-test et Pos-test

\begin{tabular}{ccc}
\hline \multirow{2}{*}{ Statistique } & \multicolumn{2}{c}{ Classe } \\
\cline { 2 - 3 } & Prétest & Prétest \\
\hline $\begin{array}{c}\text { Score le plus } \\
\text { élevé }\end{array}$ & 77,00 & 100,00 \\
$\begin{array}{c}\text { Score la plus } \\
\text { basse }\end{array}$ & 30,00 & 67,00 \\
Médian & 49,49 & 85,09 \\
Écart-type & 11,51 & 8,27 \\
\hline
\end{tabular}

Sur la base du tableau 2, la valeur moyenne du score du prétest est de 45,34 avec la valeur la plus élevée de 77,00 et la valeur la plus faible de 30,0 et l'écart type de 11,51 , tandis que pour le score post-test moyen de 85,09 avec la valeur la plus élevée de 100 et la valeur la plus faible 67,00 et écart-type de 8,27.

2. Exigences relatives à l'analyse des données de test

a. Test de normalité des données

Le test de normalité des données peut être utilisé pour voir si les données pré-test et post-test des étudiants sont normalement distribuées. Pour tester la normalité des données sur les capacités des élèves, selon Sudjana (2003), cela peut être fait en utilisant le test de Liliefors.

À partir des résultats du calcul des données de prétest pour la classe, on obtient que le Lcount soit respectivement de 0,151 et 0,119, tandis que la table $\mathrm{L}=0,190$. Sur la base des critères de comptage $\mathrm{L}<$ table $\mathrm{L}$, les données de pré-test pour la classe expérimentale et la classe témoin étaient normalement distribuées.

De même, pour les données post-test pour la classe le Lcount a été obtenu, à savoir 0,132 et 0,138 , tandis que la table $\mathrm{L}=0,161$. Sur la base des critères $\mathrm{L}$ count $<\mathrm{L}$ table, les données post-test de la classe expérimentale et de la classe témoin sont normalement distribuées. Peut être vu dans le tableau 4:17 Tableau 3. Résultats du test de normalité des données prétest et post-test
Tableau 3. Résultats Du Test De Normalité des Données Prétest Et Post-Test

\begin{tabular}{cccccc}
\hline Données & Classe & $\mathrm{L}_{\text {compter }}$ & Importance & $\mathrm{L}_{\text {tableau }}$ & $\alpha$ \\
\hline \multirow{2}{*}{ Classe } & Prétest & 0,203 & 0,175 & 0,176 & 0,05 \\
& Posttest & 0,131 & 0,002 & 0,176 & 0,05 \\
\hline
\end{tabular}

Basé sur le tableau 4.17 et le tableau 4.11 , on peut voir que dans les deux classes $\mathrm{L}$ compte $<$ L table, on peut donc conclure que les données de prétest et de post-test pour les deux classes sont normalement distribuées au niveau de signification $(\alpha=0,05)$.

\section{CONCLUSION}

À partir des résultats de l'analyse des données, le processus d'apprentissage de français en utilissant le methode de français basé sur google classroom en Classe XI à SMA Negeri 21 Medan. La phase d'Analyse, la réponse de 20 lycéens sur le média. Et puis phase de Conception. Ensuite phase de dévelopemment ,comment le processus de création de média. Puis Phse de Mise en oeuvr, C'est- à- dire comment les étaphes pour entrer dans le média, on commence avec l'affichage de signe, L'affichez la page de home sur Google Classroom. Ensuite phase d'évaluations résultats de la validation pars des experts en matériaux est la moyenne 77,95 \% (bien) et des experts des médias pédagogiques sur le Media d'apprentissage de Google Classroom en français est 77, $72 \%$, (bien). Ensuite description de données des résultats des tests d'utilisation se compose Données sur les résultats du test d'utilisation des médias par l'enseignant. La moyenne est $81.06 \%$ (très bien). Données sur les résultats de l'utilisation des médias par les lycéens se compose le test individuel est la moyenne 87, $3 \%$ (très bien), le test en petit groupe est la moyenne $89 \%$ (très bien), le test d'essai limité est la moyenne 91,7 \% (très bien). Ensuite le résultat des tests d'efficacité du média. Analyse des résultats des données de recherche. Cette recherche a fait à SMA Negeri 21 Medan avec une Classe. Dans la classe se compose 32 lycéens, le résultat prétest et postes, la valeur moyenne du score du pré-test est de 45,34 avec la valeur la plus élevée de 77,00 et la valeur la plus faible de 30,0 et l'écart type de 11,51 , tandis que pour le score post-test moyen de 85,09 avec la 
valeur la plus élevée de 100 et la valeur la plus faible 67,00 et écart-type de 8,27. Et puis le test de normalité, À partir des résultats du calcul des données de pré-test pour la classe, on obtient que le Lcount est respectivement de 0,151 et 0,119 , tandis que la table $\mathrm{L}=$ 0,190 . Sur la base des critères de comptage $L$ <table L, les données de pré-test pour la classe expérimentale et la classe témoin étaient normalement distribuées. De même, pour les données post-test pour la classe le Lcount a été obtenu, à savoir 0,132 et 0,138 , tandis que la table $\mathrm{L}=0,161$. Sur la base des critères L count $<\mathrm{L}$ table, les données posttest de la classe expérimentale et de la classe témoin sont normalement distribuées. Ensuite le test d'hypothèses, on peut voir que dans cette classe $t$ compte $\geq t$ table de sorte qu'il peut être conclu que l'apprentissage en utilisant la méthode française basée sur Google Classroom est meilleur au niveau de signification $(\alpha=0,05)$. En plus, l'utilisation des média d'apprentissage de Google Classroom peut améliorer efficacement les résultats d'apprentissage. La valeur moyenne des résultats d'apprentissage avec l'apprentissage utilisant les média d'apprentissage de Google Classroom a obtenu le score le plus élevé au post-test de 100,00 , meilleur que le score le plus élevé au prétest de 77,00 .

\section{BIBLIOGRAPHIE}

Akhtar, Ahsan, dkk. 2008. Use of Media for Effective Instruction Its Importance: Some Consideration. Pakistan : university of the punjab.

Arsyad Azhar. 2013. Media Pembelajaran. Jakarta : Raja Grafindo persada.
Ariesto, Hadi, Sutopo,2003, Multimedia Interaktif dan Flash, PT. Graha Ilmu Yogyakarta.

Borg, W \& V.Gall, MD. 1983 Educational Research an introduction (And ed) New York \& London.

Brandie, Key Ann Taylor dan Jerry Wills. 2000. Constructivist Instructional

Depdiknas, 2008. Panduan Pengembangan Bahan Ajar, Jakarta ; Direktorat.

Jenderal Pendidikan Dasar dan Menenngah Direktorat Pembinaan Sekolah menengah Atas.

Eviyanti, Evi, 2018. Schoology Implementation in the Teaching of Production Orale Pré Élémentaire.

Hardiyana, Andri. 2015. Implementasi Google Classroom sebagai Alternatif dalam Meningkatkan Mutu Pembelajaran di Sekolah. Karya Tulis Ilmiah, Cirebon: SMA Negeri 1 Losari.

Hamalik,Oemar, 2003, Kurikulum dan Pembelajaran, Jakarta . Bumi Aksara.

L. Anang Setiyo W, 2013, Perancangan Elearning dengan Menggunakan Learning Management System (LMS), Widya Warta No. 02 Tahun XXXV II/ Juli 2013 ISSN 0854-1981.

Sanjaya, Wina. 2009. Strategi Pembelajaran Berorientasi Standar Proses Pendidikan . Jakarta : Prenada .Media Grup.

Sudjana, Nana. (2004). Penilaian Hasil Proses Belajar Mengajar. Bandung : Rosdakarya

Sudjana. 2002. Metoda Statistika. Bandung: Taristo.

Sugiyono. 2015. Metode Penelitian Pendidikan. Bandung : Alpabeta Bandung.

Sugiyono. (2010). Metode Penelitian Kuantitatif, Kualitatif, dan R\&D. Bandung: Alfabeta. 